\title{
Análisis cuasi-dinámico de la inclusión de generación distribuida en sistemas eléctricos de potencia, caso de estudio: sistema IEEE de 30 nodos
}

\section{Quasi-dynamic analysis of the inclusion of generation distributed in power systems, study case: IEEE 30 nodes system}

\author{
Luis Felipe Gaitán-Cubides¹, Juan David Gómez-Ariza² ${ }^{2}$ Edwin Rivas-Trujillo $^{3}$ \\ ${ }^{1}$ Grupo de Compatibilidad e Interferencia Electromagnética (GCEM), Ingeniería Eléctrica, Universidad Distrital Francisco José de \\ Caldas, Colombia. Email: luis.f.g@ieee.org \\ ${ }^{2}$ Grupo de Compatibilidad e Interferencia Electromagnética (GCEM), Ingeniería Eléctrica, Universidad Distrital Francisco José \\ de Caldas, Colombia. Email: juan.d.g@ieee.org \\ ${ }^{3}$ Grupo de Compatibilidad e Interferencia Electromagnética (GCEM), Ingeniería Eléctrica, Universidad Distrital Francisco José \\ de Caldas, Colombia. Email: erivas@udistrital.edu.co
}

Recibido: octubre 10, 2017. Aceptado: diciembre 14, 2017. Versión final: febrero 24, 2018.

\begin{abstract}
Resumen
Los últimos avances en los sistemas de generación distribuida (GD) han abierto un nuevo escenario en las estrategias de planeación de los sistemas eléctricos. Toma gran importancia determinar los impactos económico y técnico que acarrean la instalación y la puesta en operación de GD en aquellos lugares del sistema de potencia donde antes solo estaban instaladas. En esta investigación, un proceso de optimización del despacho económico permite encontrar los valores óptimos de potencia a los que deben despachar todas las unidades del sistema IEEE de 30 nodos, modificado con nodos de GD, y procurar que se minimicen los costos de generación en las unidades convencionales. Los resultados de este proceso de optimización se implementan en unas 'simulaciones cuasidinámicas' que permiten identificar y analizar los parámetros que varían en el sistema a lo largo del tiempo, además de dimensionar los valores de pérdidas de potencia en las líneas y en los transformadores del sistema.
\end{abstract}

Palabras clave: generación distribuida; optimización por enjambre de partículas; simulación cuasidinámica.

\begin{abstract}
The latest advances in Distributed Generation (GD) systems have opened a new stage in planning strategies for electrical systems. It is of great importance to determine the economic and technical impact of the installation and commissioning of GD in those parts of the power system where they were previously installed. In this research, the process of optimization of the economic dispatch allows to find the optimum power values to which all the units of the 30-node IEEE system, modified with GD nodes, must be dispatched, trying to minimize generation costs in conventional units. The results of this optimization process are implemented in a "Quasi-Dynamic Simulation" that allows to identify and analyze the parameters that vary in the system over time, in addition to dimensioning the values of power losses in the lines and transformers of the system.
\end{abstract}

Keywords: distributed generation; particle swarm optimization; quasi-dynamic simulation.

ISSN impreso: 1657 - 4583, ISSN en línea: 2145 - 8456

Este artículo puede compartirse bajo la licencia CC BY-ND 4.0 y se referencia usando el siguiente formato: L. Gaitán, J. Gómez, E. Rivas, "Análisis cuasi-dinámico de la inclusión de generación distribuida en sistemas eléctricos de potencia, caso de estudio: sistema IEEE de 30 nodos," Rev. UIS Ing., vol. 17, no. 2, pp. 41-54, 2018. Doi: https://doi.org/10.18273/revuin.v17n2-2018004 


\section{Introducción}

El término generación distribuida (GD) no tiene una definición única, depende de la legislación de cada país [1] [2]. Para el caso colombiano, [3] define la GD como la producción de energía eléctrica cerca de los centros de consumo, conectada a un sistema de distribución local (SDL). La capacidad de la generación distribuida se definirá en función de la capacidad del sistema en donde se va a conectar, según los términos del código de conexión y las demás disposiciones que la Comisión Reguladora de Energía y Gas (Creg) defina para tal fin.

La necesidad de un sistema eléctrico más flexible, la implementación de estrategias de uso racional de la energía y los cambios en las políticas energéticas han aportado a la aparición de sistemas de generación distribuida. Este nuevo escenario de generación de electricidad proyecta una serie de cambios en las estrategias de planeación y operación de las redes eléctricas el mundo. Al presentarse cambios en los elementos constitutivos del sistema, es necesario observar las consecuencias económicas y técnicas que se derivan por su inclusión. Por tal motivo se requiere realizar los estudios de flujo de carga y optimización del sistema antes y después de la inclusión de la generación distribuida.

Determinar los cambios en las variables que miden el comportamiento del sistema eléctrico de potencia requiere un análisis particular, ya que identificar el impacto de la conexión de estos generadores permitiría conocer cuán beneficioso es para la red eléctrica y los usuarios la inclusión de aquellos en el sistema.

Es conveniente realizar los análisis de flujo de carga a través de simulaciones 'cuasidinámicas'. Estas simulaciones en esencia son flujos de carga a lo largo de un periodo de 24 horas en los diferentes nodos de un sistema eléctrico de potencia, que permiten identificar y analizar los parámetros que varían en él a lo largo del tiempo, además de dimensionar los valores de pérdidas de potencia en las líneas y en sus transformadores.

\section{Simulación del sistema IEEE de 30 nodos con generación distribuida}

El sistema IEEE de 30 nodos corresponde a una red eléctrica del medio este de los Estados Unidos (Nueva Inglaterra). Tiene 6 generadores, 41 líneas de transmisión, 21 nodos de carga, 7 transformadores y una demanda del sistema de 283,4 MW y 126,2 MVAr [4] [5]. Este sistema de potencia se simuló en DigSilent ${ }^{\circledR}$ Powerfactory Versión 15.1. replicando los datos de la simulación que se encuentra en [6].

\subsection{Pérdidas en los transformadores}

El sistema IEEE de 30 nodos es concebido originalmente sin pérdidas en sus devanados. En los escenarios de simulación de los casos de estudio se ajustó a un valor de pérdida que se determinó de acuerdo con la «Norma técnica colombiana NTC 819 electrotecnia. Transformadores trifásicos autorrefrigerados y sumergidos en líquido. Corriente sin carga, pérdidas y tensión de cortocircuito» [7].

En la sección 5 de esta norma se referencia la ecuación como una ecuación válida para determinar el valor de pérdidas con carga presentes en los transformadores. Se debe tener en cuenta que esta norma solo menciona transformadores hasta de $10 \mathrm{MVA}$, y los transformadores presentes en ambos sistemas IEEE son de 100 MVA; sin embargo, es una buena aproximación a un valor de pérdidas que podría llegar a tener un transformador. En las simulaciones se ajusta un nivel de $76 \mathrm{KW}$, como las pérdidas presentes en el cobre de los devanados.

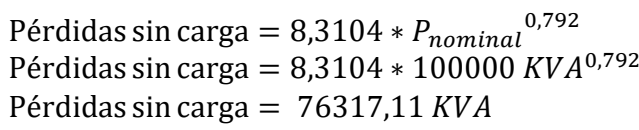

Pérdidas sin carga $=76317,11 \mathrm{KVA}$

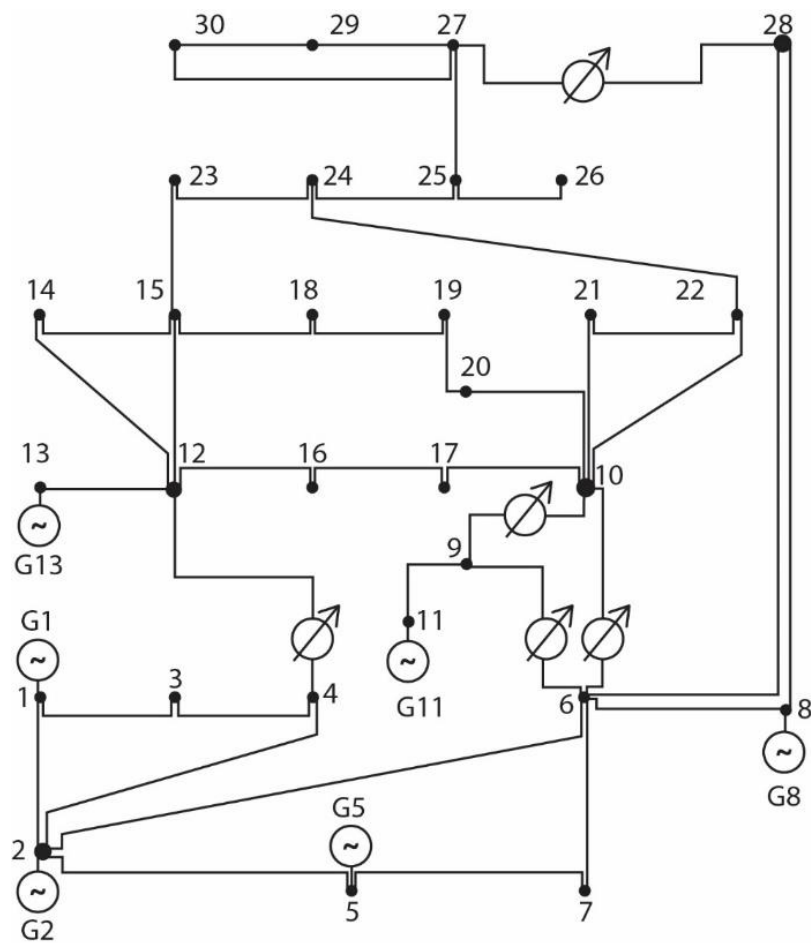

Figura 1. Esquema del sistema IEEE de 30 nodos original usado en el caso de estudio. Fuente: elaboración propia.

\subsection{Inclusión de curvas de demanda en las cargas}

Para cada una de las cargas presentes en el sistema se modeló una curva de demanda diaria. Las curvas de carga 
diaria indican las características de la carga en el sistema, sean estas predominantemente residenciales, comerciales o industriales y de la forma en que se combinan con el fin de producir los picos.

Se simuló la curva industrial en cada una de las cargas, ya que esta clase de clientes son los que tienen la capacidad económica de adquirir y poner en marcha los equipos necesarios para la generación de electricidad de manera independiente, y aprovechar los eventuales descuentos y auxilios que les brinde el Gobierno nacional. Los valores de esta curva fueron tomadas de [8].

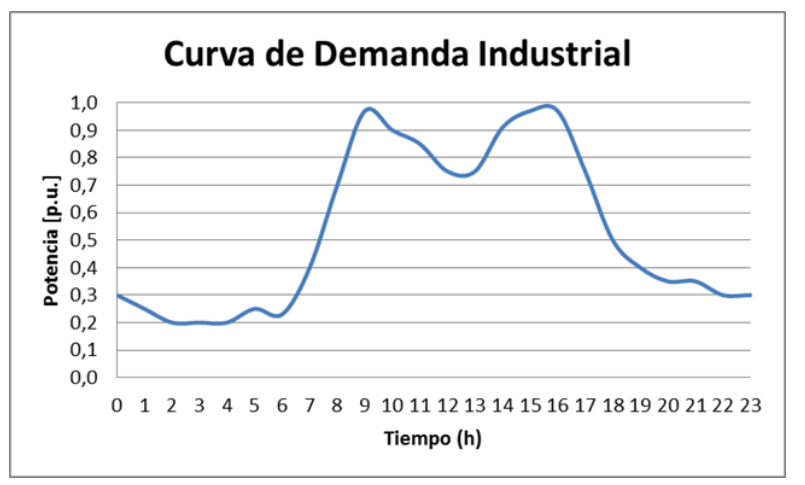

Figura 2. Curva de demanda industrial del sector eléctrico colombiano. Fuente: [8].

\subsection{Modelamiento de los sistemas de GD}

Para simular los generadores alternativos en el sistema de potencia se adiciona, en los nodos trifásicos donde se encuentran cargas tipo spot, dos nuevos barrajes a través de un breaker/switch. En uno de estos nuevos barrajes se ubica la carga que tenía inicialmente el nodo original y en el otro se encuentra ubicado un transformador que eleva el nivel de tensión proveniente de un generador.

Tabla 1. Capacidad de los generadores añadidos al sistema IEEE de 30 nodos.

\begin{tabular}{|l|l|}
\hline Generador & Potencia activa $[\mathbf{M W}]$ \\
\hline Generador 23 & 3,2 \\
\hline Generador 24 & 8,7 \\
\hline Generador 26 & 3,5 \\
\hline Generador 29 & 2,4 \\
\hline Generador 30 & 10,6 \\
\hline
\end{tabular}

\subsubsection{Modelamiento del generador alternativo}

El generador alternativo se configura a partir de la herramienta static generator del software DigSilent ${ }^{\circledR}$ Powerfactory, el cual modela el comportamiento de la mayoría de generadores de energía eléctrica a partir de fuentes renovables que se conectan a la red a través de un inversor [9] [10] [23].

El valor del factor de potencia que van a tener estos generadores es de 0,9. Para determinar el valor de potencia activa $y$ de potencia aparente de los generadores, se toma como referencia el valor de la potencia activa de la carga tipo spot que estaba originalmente en el sistema de potencia. De tal manera que los valores de los generadores alternativos se calculan con la relación entre este valor y el factor de potencia, tal como se relaciona en la ecuación

$$
P=S \cdot \text { Factor de potencia }
$$

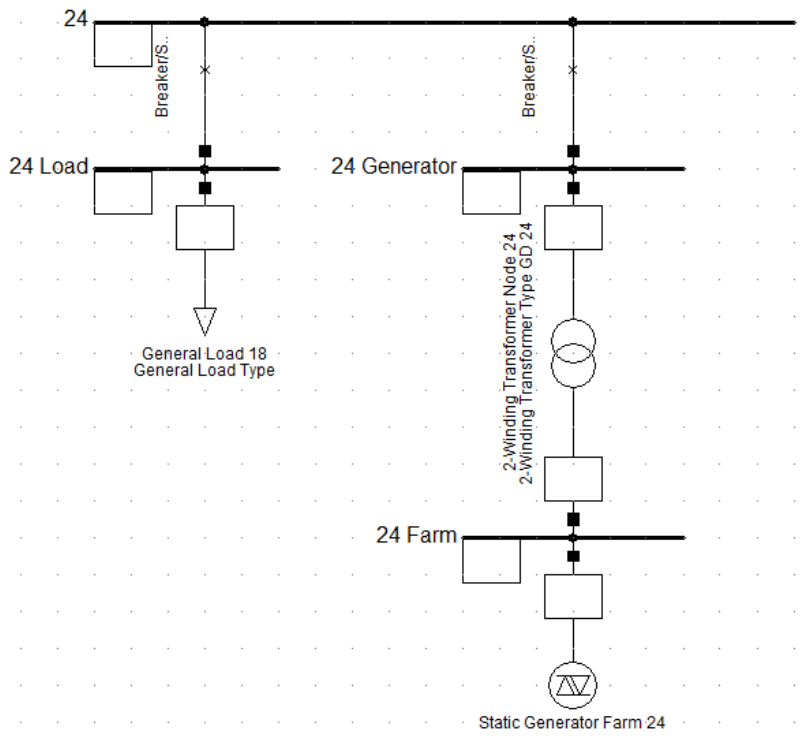

Figura 3. Esquema de los elementos adicionales del sistema IEEE de 30 nodos modificado con fuentes de generación distribuida usado en el caso de estudio simulado en DigSilent ${ }^{\circledR}$ Powerfactory. Fuente: elaboración propia. 


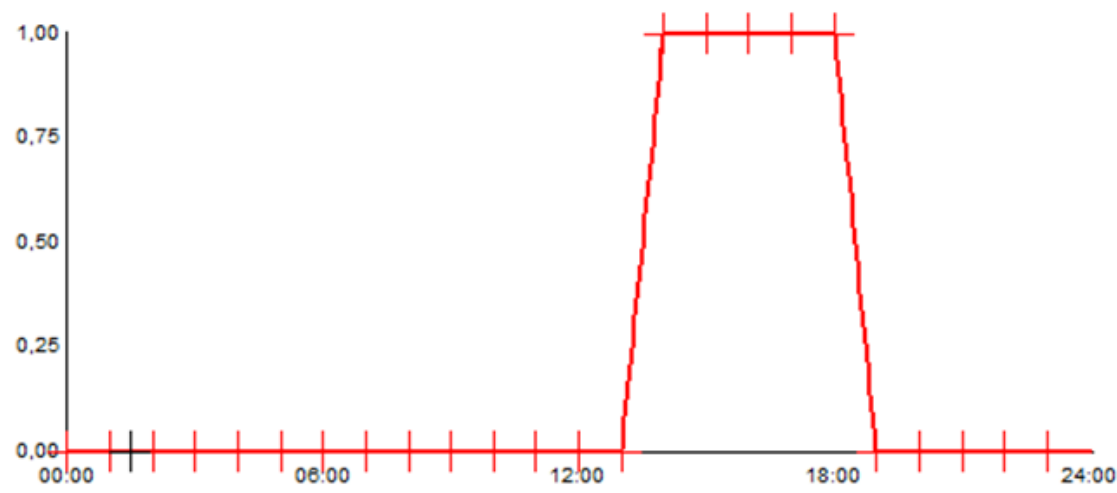

Figura 4. Curva de despacho de las unidades de generación distribuida para la simulación en DigSilent@ Powerfactory. Fuente: elaboración propia.

Estos generadores alternativos se ajustan de tal manera que inician su despacho de potencia a las 14:00:00 horas del día y finaliza a las 18:00:00, atendiendo el segundo pico de la curva de demanda típico de carga industrial.

\subsubsection{Modelamiento de los transformadores para los generadores alternativos}

Para el nodo donde se encuentra el generador alternativo se modela también un transformador cuya función es adecuar el nivel de tensión presente a la salida del generador alternativo (nodo farm) y el nivel de tensión presente en el sistema de potencia original (nodo generator).

El nivel de tensión del nuevo generador es de 690 voltios, este valor se incluye basándose en ejemplos de sistemas con generación alternativa ofrecidos por DigSilent ${ }^{\circledR}$ Powerfactory. En adición, otra característica incluida en los nuevos transformadores presentes en el sistema es que las pérdidas de este serán de $3 \%$ y que su conexión en Estrella Neutro Cero (YN-YN-0).

Los demás valores se dejan por defecto tal como los genera el software.

\subsection{Escenarios de simulación}

Para estudiar e identificar el impacto de estos nuevos generadores en los sistemas de potencia se ajustaron las simulaciones como se describe a continuación.

\subsubsection{Escenario convencional}

En este escenario de operación los sistemas de potencia solo tienen presentes las cargas y los generadores convencionales que ofrecen los autores. De la misma manera, en este escenario, solo están activados los breaker/switch que conectan los nodos load, que tienen asociados las cargas tipo spot presentes en el sistema de potencia original.

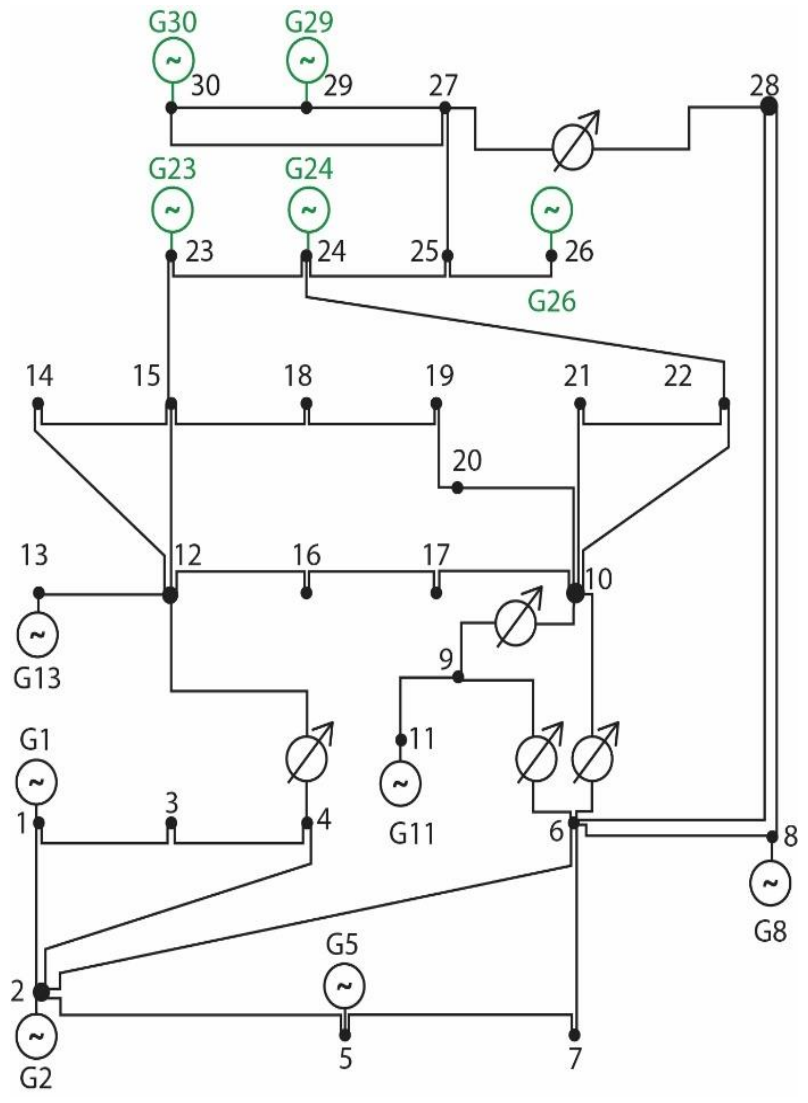

Figura 5. Esquema del sistema IEEE de 30 nodos modificado con fuentes de generación distribuida usado en el caso de estudio. Fuente: elaboración propia.

\subsubsection{Escenario generación distribuida}

En este escenario de operación están activadas, al mismo tiempo, todas las cargas y los generadores incluidos en el 
escenario alternativo. En este escenario de operación están activados los breaker/switch que conectan tanto los nodos load como los nodos generator.

Según [11], es de gran importancia en cualquier proceso de simulación, control o supervisión de un sistema eléctrico que haya similitud entre el sistema real y el modelo, y esta debe ser tal que los resultados que se obtengan sean lo más precisos posibles.

Tabla 2. Tipo de nodos en el sistema IEEE de 30 nodos en los escenarios convencional y generación distribuida.

\begin{tabular}{|l|l|l|}
\hline Nodo & Esc. convencional & Esc. generación dist. \\
\hline $\mathbf{1}$ & Generación & Generación \\
\hline $\mathbf{2}$ & Generación - carga & Generación - carga \\
\hline $\mathbf{3}$ & Carga & Carga \\
\hline $\mathbf{4}$ & Carga & Carga \\
\hline $\mathbf{5}$ & Generación - carga & Generación - carga \\
\hline $\mathbf{6}$ & Subestación & Subestación \\
\hline $\mathbf{7}$ & Carga & Carga \\
\hline $\mathbf{8}$ & Generación - carga & Generación - carga \\
\hline $\mathbf{9}$ & Subestación & Subestación \\
\hline $\mathbf{1 0}$ & Subestación - carga & Subestación - carga \\
\hline $\mathbf{1 1}$ & Generación & Generación \\
\hline $\mathbf{1 2}$ & Subestación - carga & Subestación - carga \\
\hline $\mathbf{1 3}$ & Generación & Generación \\
\hline $\mathbf{1 4}$ & Carga & Carga \\
\hline $\mathbf{1 5}$ & Carga & Carga \\
\hline $\mathbf{1 6}$ & Carga & Carga \\
\hline $\mathbf{1 7}$ & Carga & Carga \\
\hline $\mathbf{1 8}$ & Carga & Carga \\
\hline $\mathbf{1 9}$ & Carga & Carga \\
\hline $\mathbf{2 0}$ & Carga & Carga \\
\hline $\mathbf{2 1}$ & Carga & Carga \\
\hline $\mathbf{2 2}$ & Paso & Paso \\
\hline $\mathbf{2 3}$ & Carga & Generación - carga \\
\hline $\mathbf{2 4}$ & Carga & Generación - carga \\
\hline $\mathbf{2 5}$ & Paso & Paso \\
\hline $\mathbf{2 6}$ & Carga & Generación - carga \\
\hline $\mathbf{2 7}$ & Paso & Paso \\
\hline $\mathbf{2 8}$ & Subestación & Subestación \\
\hline $\mathbf{2 9}$ & Carga & Generación - carga \\
\hline $\mathbf{3 0}$ & Carga & Generación - carga \\
\hline & & \\
\hline & & \\
\hline
\end{tabular}

Ese mismo autor menciona que al agregar una fuente de generación a la red de distribución se debe garantizar que la máxima inyección de potencia de esta fuente durante el análisis sea la misma potencia máxima real que podría entregar, o que los parámetros de cualquier elemento del sistema tengan un valor pertinente de acuerdo con el nivel de tensión o tipo de red. Por tal razon, a lo largo de la simuación las fuentes de GD están entregando el $100 \%$ de su capacidad instalada.

\section{Optimización del despacho económico}

Previo a ejecutar la simulación cuasidinámica, se realiza la optimización del despacho económico en los dos escenarios de simulación. Este despacho se realiza a partir de la premisa que las fuentes de generación distribuida entregarán el total de su generación disponible, en el pico más alto de la curva de demanda energética.

Para realizar el proceso de optimización, se selecciona el método de enjambre de partículas, conocido como particle swarm optimization (PSO). Este método fue desarrollado por James Kennedy y Russell Eberhart en 1995, en él introducen el concepto de optimización de funciones no lineales [12] [13] [22].

El objetivo de aplicar esta metodología es lograr la optimización del costo de combustible de los generadores convencionales del sistema, que para el caso de estudio son los generadores que vienen originalmente con el sistema, G1, G2, G5, G8, G11 y G13. Esta función de optimización está sujeta a las restricciones de tensión y de potencia en cada uno de los nodos utilizando el método de optimización por enjambre de partículas PSO.

El proceso de optimización se realizó utilizando el método de optimización por enjambre de partículas, mediante el software Matlab y con base en particle swarm optimization toolbox [14]. Este toolbox fue desarrollado por Brian Birge en 2005 y recibió una actualización en 2006, y, a pesar de no haber recibido ninguna actualización en 10 años, sigue siendo un referente en varios trabajos de investigación [15] [16] [17] [18] [19].

Para solucionar el problema, [20] plantea las siguientes ecuaciones:

1. Función de costo total del combustible:

$$
f\left(P_{G}\right)=\sum_{i=1}^{N_{g}} f_{i}\left(P_{G i}\right)
$$

2. Potencia activa con la función cuadrática de costo

$$
f_{i}\left(P_{G i}\right)=a_{i} P_{G i}^{2}+b_{i} P_{G i}+c_{i}
$$

Donde:

$f\left(P_{G}\right)$ es el costo de la producción total en $\$ / \mathrm{hr}$

$f_{i}\left(P_{G i}\right)$ es la función costo de combustible de unidad i en $\$ / \mathrm{hr}$

$a_{i} b_{i} c_{i}$ son los coeficientes del costo de combustible de unidad i

$P_{G i}$ es la potencia real entregada de unidad i en MW.

Este método de optimización de partículas se implementó en el sistema IEEE de 30 nodos para obtener la 
minimización de los costos de generación. Debido al carácter aleatorio del algoritmo, se realizaron 100 simulaciones en cada sistema y se calculó el promedio de cada uno de los valores.

Para lograr que los generadores alternativos siempre estuvieran a plena carga se ajustaron los valores de los coeficientes a, b y c. De tal manera que, al realizar el proceso de optimización, el algoritmo garantizaba que estos generadores estuvieran despachando al $100 \%$ de su capacidad, y esto hacía que el despacho de los generadores convencionales se ajustara a esa condición.

Los coeficientes utilizados en los generadores alternativos son un acercamiento general a su modelamiento, por lo tanto, si se quisiera conocer los resultados de inclusión de un tipo de generación particular, es necesario realizar el modelamiento, la modificación y la adaptación de las ecuaciones actuales usadas en el cálculo de costos de generación. En lo anterior se tuvo en cuenta que cada tipo de generación funciona de manera diferente, y, en consecuencia, los valores de los coeficientes pueden variar de un tipo a otro.

Los resultados de esta optimización se resumen en la tabla 3. Estos valores son aplicados en la simulación del sistema IEEE de 30 nodos realizada en DigSilent ${ }^{\circledR}$ Powerfactory.

Tabla 3. Tipo de nodos en el sistema IEEE de 30 nodos en los escenarios convencional y generación distribuida.

\begin{tabular}{|l|l|l|l|}
\hline \multicolumn{4}{|c|}{ Valores de potencia de los generadores } \\
\hline Esc. convencional & \multicolumn{2}{|c|}{ Esc. generación distribuida } \\
\hline Gen & P $[\mathbf{M W}]$ & Gen & P $[\mathbf{M W}]$ \\
\hline G1 & 173,3735 & G1 & 128,4042 \\
\hline G2 & 48,0005 & G2 & 35,9846 \\
\hline G5 & 21,2204 & G5 & 20,6909 \\
\hline G8 & 24,3824 & G8 & 33,3931 \\
\hline G11 & 12,4963 & G11 & 22,5782 \\
\hline G13 & 12,0000 & G13 & 18,3961 \\
\hline & & G23 & 4,5005 \\
\cline { 3 - 4 } & & G24 & 12,3314 \\
\cline { 3 - 4 } & G26 & 4,9245 \\
\cline { 3 - 4 } & & G29 & 3,4279 \\
\cline { 3 - 4 } & & G30 & 15,0647 \\
\hline
\end{tabular}

\section{Simulacion 'cuasidinámica'}

El cálculo del flujo de carga limita el análisis de los sistemas de potencia bajo un solo conjunto de condiciones de funcionamiento. Si se quiere hacer un análisis del rendimiento del sistema en las condiciones de funcionamiento más desfavorables, el análisis de flujo de carga ofrecería un análisis parcial de la operación del sistema, debido a que por la complejidad que exige el análisis puede ser difícil entender intuitivamente cuáles escenarios operativos y estados de red causan tales condiciones [10].

Así mismo, [10] señala que para realizar un análisis a ese nivel de complejidad, se deben ejecutar varias simulaciones de flujo de carga con una gama de condiciones de funcionamiento diferentes. Esto se logra generalmente modelando los cambios en la red a lo largo del tiempo, porque la mayoría de los parámetros operacionales tienen una dependencia del tiempo. En ese orden de ideas, cuando se examina la variación del flujo de carga a lo largo del tiempo, se empieza a tener en consideración el rendimiento de la red a lo largo de horas.

Una simulación dinámica que tenga detallados todos los parámetros de control de potencia deberá ser ejecutada la misma cantidad de tiempo que se requiera simular, sin embargo, esto demandaría un gran esfuerzo computacional, y, además, implica una complejidad innecesaria si solo son de interés las condiciones de flujo de carga en estado estacionario.

En consecuencia, se considera un enfoque razonable para simular los llamados fenómenos 'cuasidinámicos' utilizar una serie de cálculos de flujo de carga dependientes del tiempo. DigSilent ${ }^{\circledR}$ Powerfactory Versión 15.1 incluye una herramienta dedicada de cálculo del flujo de carga que varía en el tiempo, llamada simulación cuasidinámica. Esta herramienta completa una serie de simulaciones de flujo de carga en un tiempo determinado, y permite que el usuario pueda seleccionar el periodo de simulación.

Para ello, la simulación cuasidinámica hace uso de los parámetros que varían en el sistema a lo largo del tiempo [10]. Para evaluar el comportamiento del sistema IEEE de 30 nodos con fuentes de generación distribuida, cuando se ha realizado un proceso de optimización del despacho económico, se grafican los perfiles de tensión, potencia activa y reactiva en un período de 24 horas de un día. En adición se presentan las pérdidas en los transformadores y las líneas en los tres escenarios. En [21] se presenta la biblioteca de elementos que posee el programa, el cual permite obtener los parámetros de tensión, potencia activa y reactiva en cada uno de sus nodos, así como los valores de corriente y las pérdidas en las líneas, los trasformadores y los demás elementos del sistema.

Para visualizar los resultados de la simulación, el sistema IEEE de 30 nodos se dividió en tres zonas, cada una con diez nodos: la zona 1 contiene los nodos 1 al 10; la zona 2 , los nodos 11 al 20, y la zona $3, \operatorname{los} \operatorname{nodos} 21$ al 30 . 


\subsection{Variación en el perfil de tensión}

La figura 6 ilustra las variaciones en el perfil de tensión. En esta zona se presenta un aumento significativo en el nivel de tensión de los nodos 21 al 30 durante los escenarios 2 y 3 . El punto donde se encuentran los cambios más significativos de tensión son aquellos donde la curva de demanda tiene su segundo pico (entre las 14:00:00 y las 17:00:00).

El nivel de tensión entre las 08:00:00 y las 11:00:00, donde está presente el primer pico de demanda, aumenta en el escenario generación distribuida, porque hay una desconexión de las cargas en los nodos 23, 24, 26, 29 y 30.

Pero se observa de nuevo la caída en el nivel de tensión en este periodo, ya que estas cargas están conectadas y demandan una cantidad considerable de potencia que no es suplida por la GD, debido a que solo está activa en el segundo pico, y se nota un aumento en el perfil de tensión, mientras estos nuevos nodos de carga están activos.
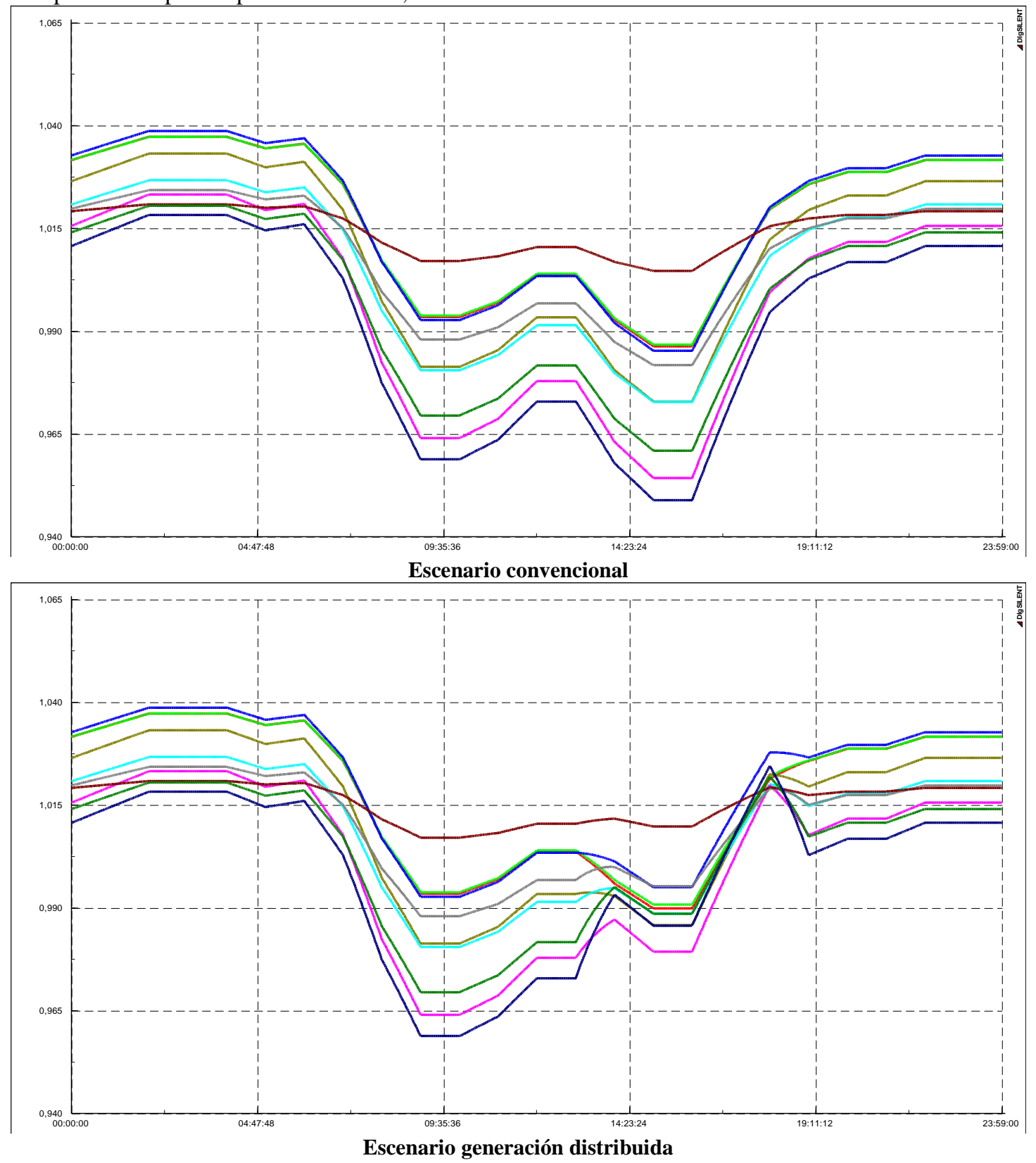

Figura 6. Variaciones en el perfil de tensión de la zona 3 (nodos 21 al 30). Fuente: elaboración propia. 

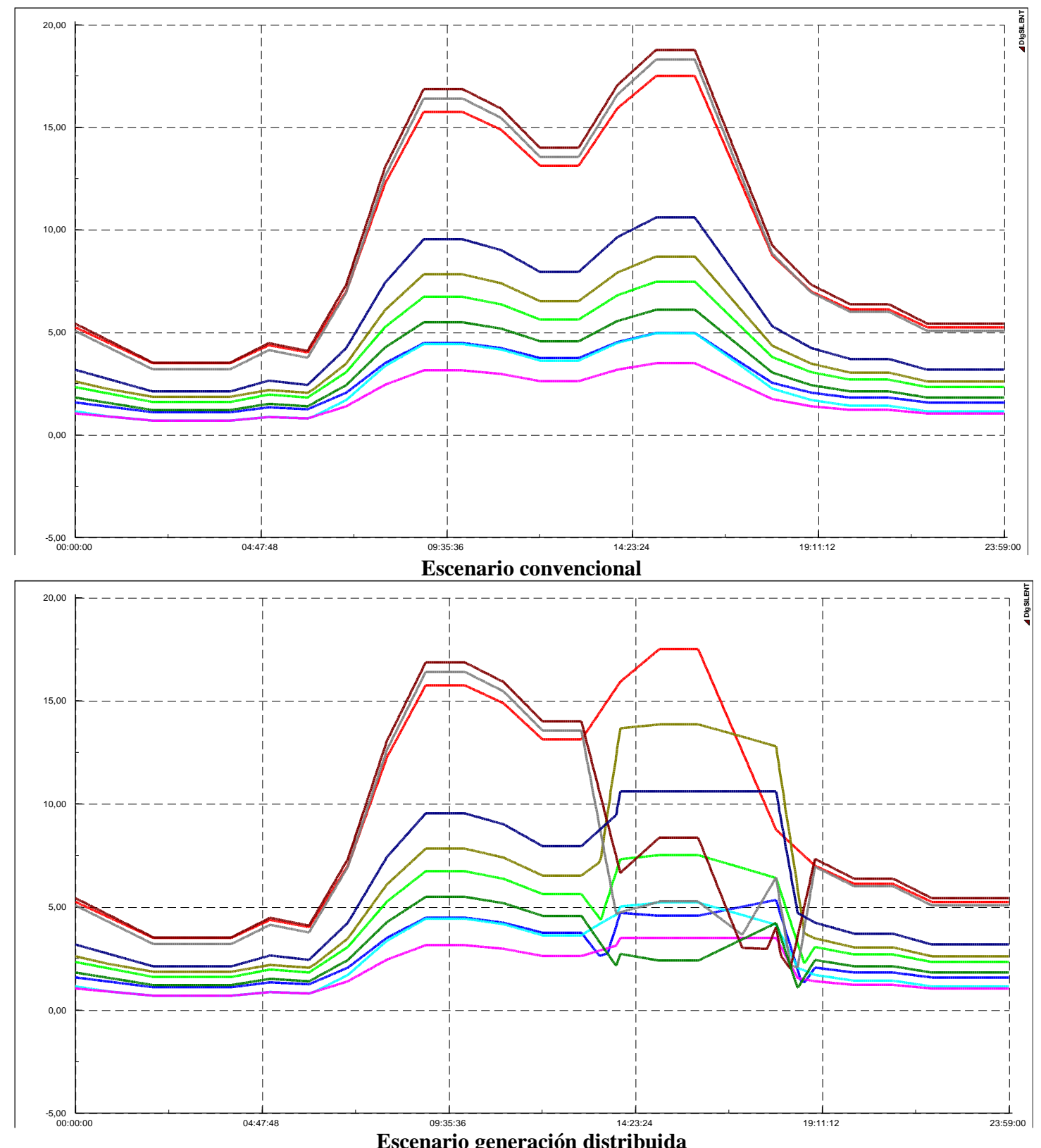

Figura 7. Variaciones en el perfil de potencia activa de la zona 3 (nodos 21 al 30). Fuente: elaboración propia.

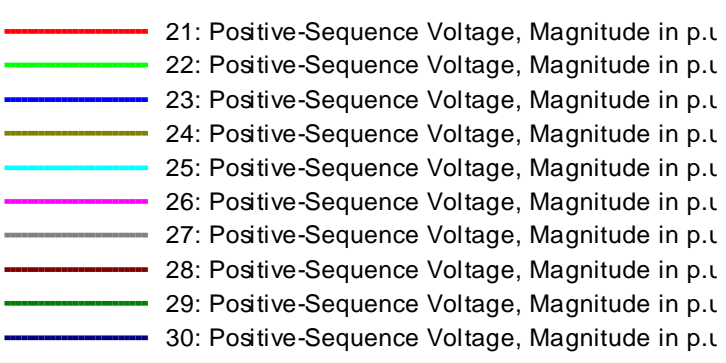

Figura 8. Código de colores para los nodos de la zona 3. Fuente: elaboración propia.

\subsection{Variación en el perfil de potencia activa}

La figura 7 ilustra las variaciones en el perfil de potencia activa. El escenario de generación distribuida, al estar presentes tanto las nuevas unidades de generación como las cargas, se observa una modificación en los niveles de potencia, pero con un compartimiento muy similar al del escenario convencional.

La diferencia está en que al entrar en operación la GD durante el segundo pico de demanda del día, la potencia demandada por las cargas está siendo suplida por los 
nuevos generadores, lo que ocasiona la caída de tensión que de observa en el nodo 28.

\subsection{Variación en el perfil de potencia reactiva}

La figura 9 ilustra las variaciones en el perfil de potencia reactiva. En el escenario convencional se identifica el comportamiento del sistema durante los picos de demanda presentes a lo largo del día.
En el escenario generación distribuida se observa cómo los picos disminuyen con la salida de algunas cargas y con la entrada en operación de los nodos de GD. Se denota una disminución en los valores máximos que se veían en el primer escenario. La entrada de la GD deja ver una disminución en los valores pico de potencia reactiva.
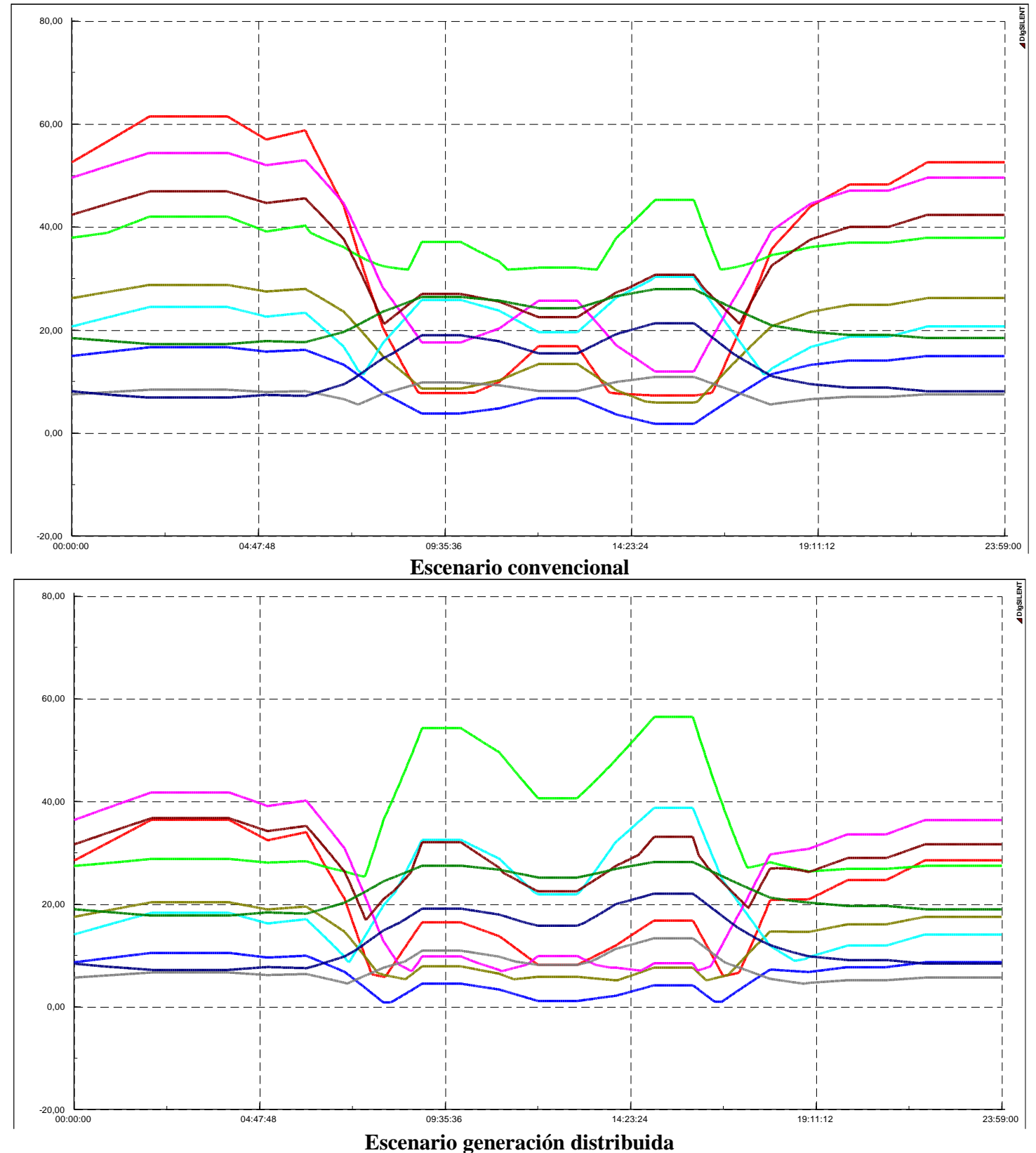

Figura 9. Variaciones en el perfil de potencia reactiva de la zona 1 (nodos 1 al 10). Fuente: elaboración propia. 


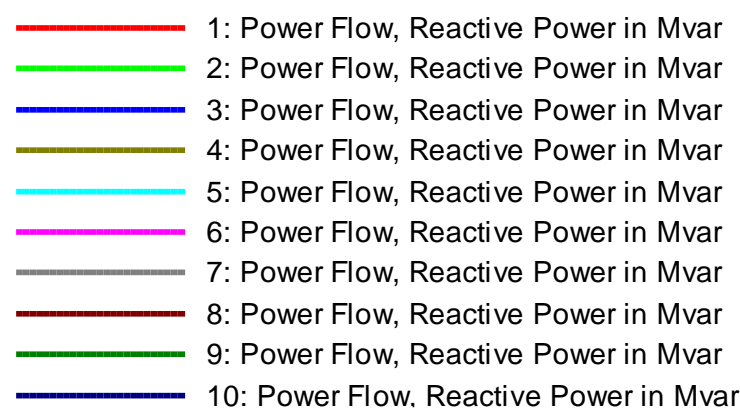

Figura 10. Código de colores para los nodos de la zona 1. Fuente: elaboración propia.

\section{Análisis de resultados}

\subsection{Variación en los transformadores}

En la tabla 4 se relaciona la diferencia porcentual entre las pérdidas presentes en los transformadores del escenario convencional y el escenario generación distribuida, donde se observa una variación de hasta un $89 \%$ en el valor de perdidas presentes en los transformadores. La diferencia porcentual entre las pérdidas de estos dos escenarios se grafica en la figura 6 .

Tabla 4. Diferencia porcentual entre las pérdidas presentes en los transformadores entre el escenario convencional y el escenario generación distribuida.

\begin{tabular}{|l|l|}
\hline \multicolumn{2}{|l|}{ Pérdidas en los transformadores sistema IEEE 30 nodos } \\
\hline Líneas & Pérdidas [kW] \\
\hline Transformador $\mathbf{1 0 / 9}$ & $-6,11 \%$ \\
\hline Transformador $\mathbf{1 2 / 1 3}$ & $3,54 \%$ \\
\hline Transformador $\mathbf{2 8 / 2 7}$ & $-70,15 \%$ \\
\hline Transformador $\mathbf{4 / 1 2}$ & $-42,93 \%$ \\
\hline Transformador $\mathbf{6 / 1 0}$ & $-38,39 \%$ \\
\hline Transformador $\mathbf{6 / 9}$ & $-59,19 \%$ \\
\hline Transformador $\mathbf{9 / 1 1}$ & $12,56 \%$ \\
\hline
\end{tabular}

5.2. Variación en las pérdidas en las líneas de transmisión.

En la tabla 5 se relaciona la diferencia porcentual entre las pérdidas en las líneas del escenario convencional y el escenario generación distribuida. En este caso, se observa cómo en las líneas 29 y 32 hay un aumento excesivo en las pérdidas de las líneas, esto se debe a que, por la ubicación de los nuevos generadores, las líneas pasan de transportar 40 a 120 amperios y 10 a 60 amperios respectivamente, lo que influye directamente en las pérdidas presentes en esas líneas.

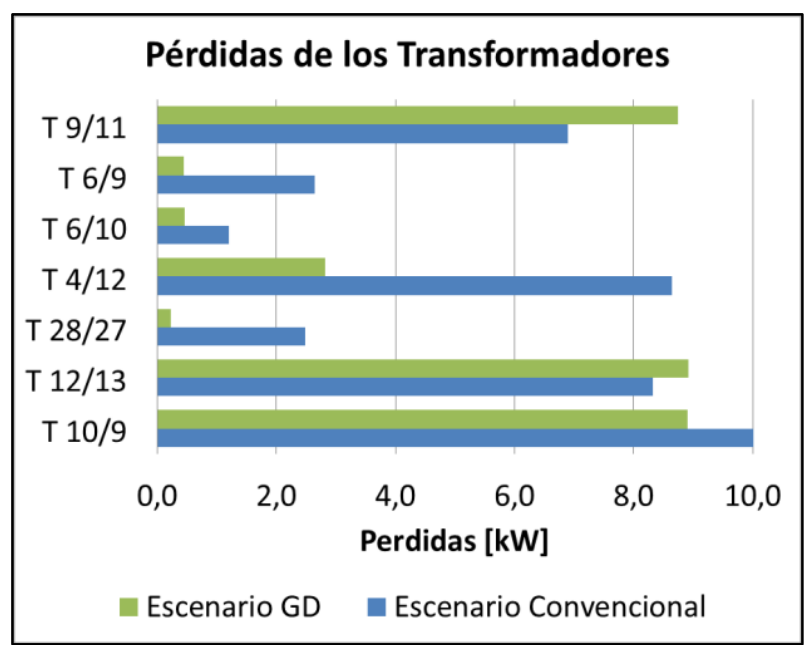

Figura 11. Valores de pérdidas en los transformadores. Fuente: elaboración propia.

Para este caso se hace necesario un estudio más profundo para determinar si estas líneas están en la capacidad de soportar ese nivel de corriente sin afectar su correcta operación. Se analiza la variación de las demás líneas sin contar las anteriormente mencionadas, y se obtienen variaciones de hasta un $97,3 \%$.

Tabla 5. Diferencia porcentual entre las pérdidas presentes en las líneas entre el escenario convencional y el escenario generación distribuida.

\begin{tabular}{|l|l|l|l|}
\hline \multicolumn{4}{|c|}{ Pérdidas en las lineas sistema IEEE 30 nodos } \\
\hline Línea & Pérdidas [kW] & Línea & \multicolumn{1}{|c|}{ Pérdidas [kW] } \\
\hline L 10 & $-71,93 \%$ & L 29 & $1298,08 \%$ \\
\hline L 11 & $7,57 \%$ & L 3 & $-73,26 \%$ \\
\hline L 12 & $-18,93 \%$ & L 30 & $-46,62 \%$ \\
\hline L 15 & $-46,25 \%$ & L 31 & $-17,57 \%$ \\
\hline L 16 a & $-36,85 \%$ & L 32 & $12415,80 \%$ \\
\hline L 16 b & $-36,85 \%$ & L 33 & $-30,82 \%$ \\
\hline L 17 & $-2,41 \%$ & L 34 & $-68,99 \%$ \\
\hline L 18 & $61,59 \%$ & L 35 & $-95,47 \%$ \\
\hline L 19 & $-18,94 \%$ & L 36 & $-93,99 \%$ \\
\hline L 2 & $-69,82 \%$ & L 37 & $-97,35 \%$ \\
\hline L 20 & $-37,63 \%$ & L 4 & $-71,50 \%$ \\
\hline L 21 & $-23,96 \%$ & L 5 & $-50,20 \%$ \\
\hline L 23 & $-44,28 \%$ & L 6 & $-71,66 \%$ \\
\hline L 24 & $-49,10 \%$ & L 7 & $-77,55 \%$ \\
\hline L 25 & $2,59 \%$ & L 8 & $3,96 \%$ \\
\hline L 26 & $-23,15 \%$ & L 9 & $-89,90 \%$ \\
\hline L 27 & $7,66 \%$ & L 1 a & $-68,74 \%$ \\
\hline L 28 & $-2,76 \%$ & L 1 b & $-68,74 \%$ \\
\hline
\end{tabular}


Análisis cuasi-dinámico de la inclusión de generación distribuida en sistemas eléctricos de potencia, caso de estudio: sistema IEEE de 30 nodos

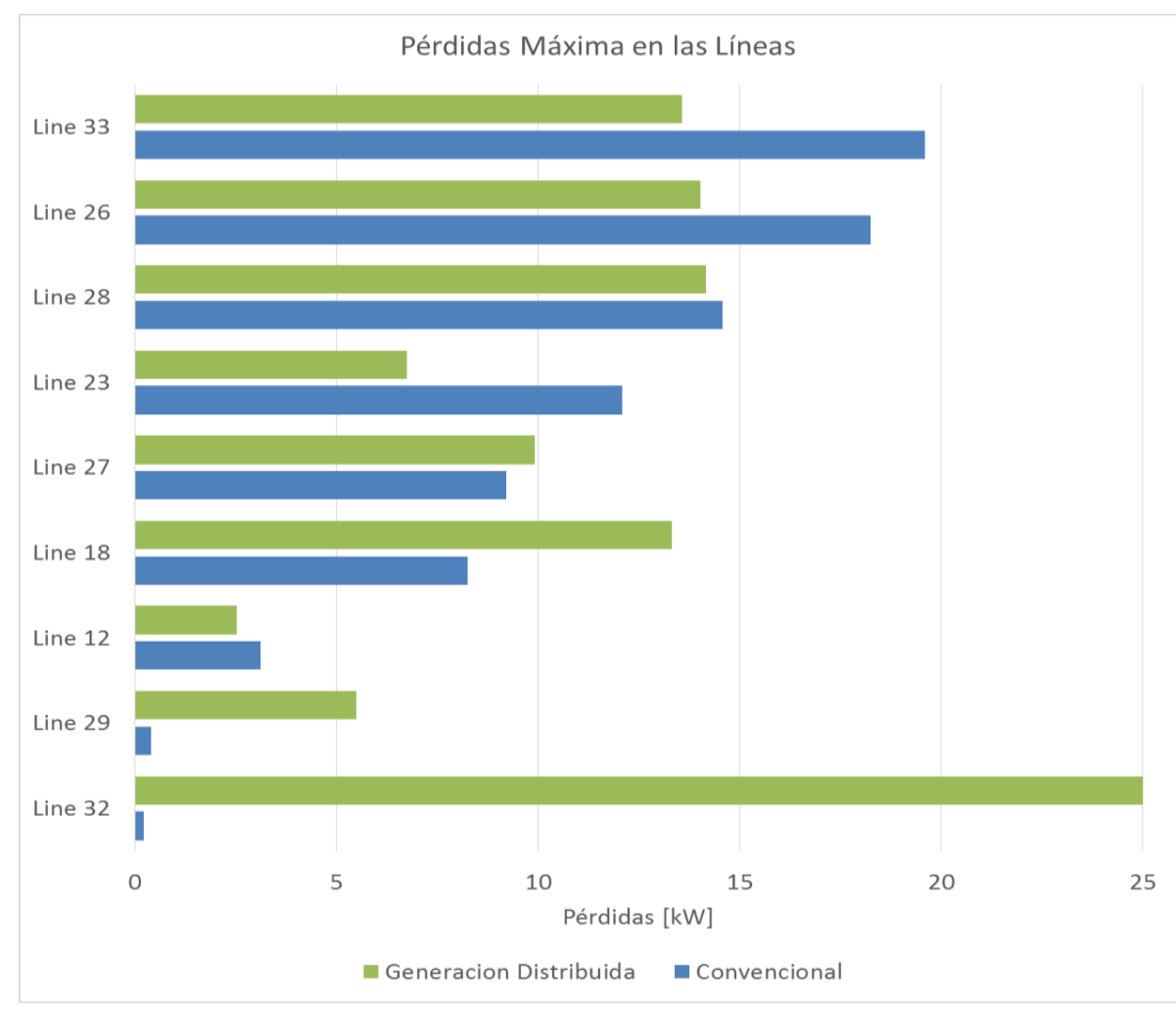

Figura 12. Valores de pérdidas en algunas líneas del sistema. Fuente: elaboración propia.

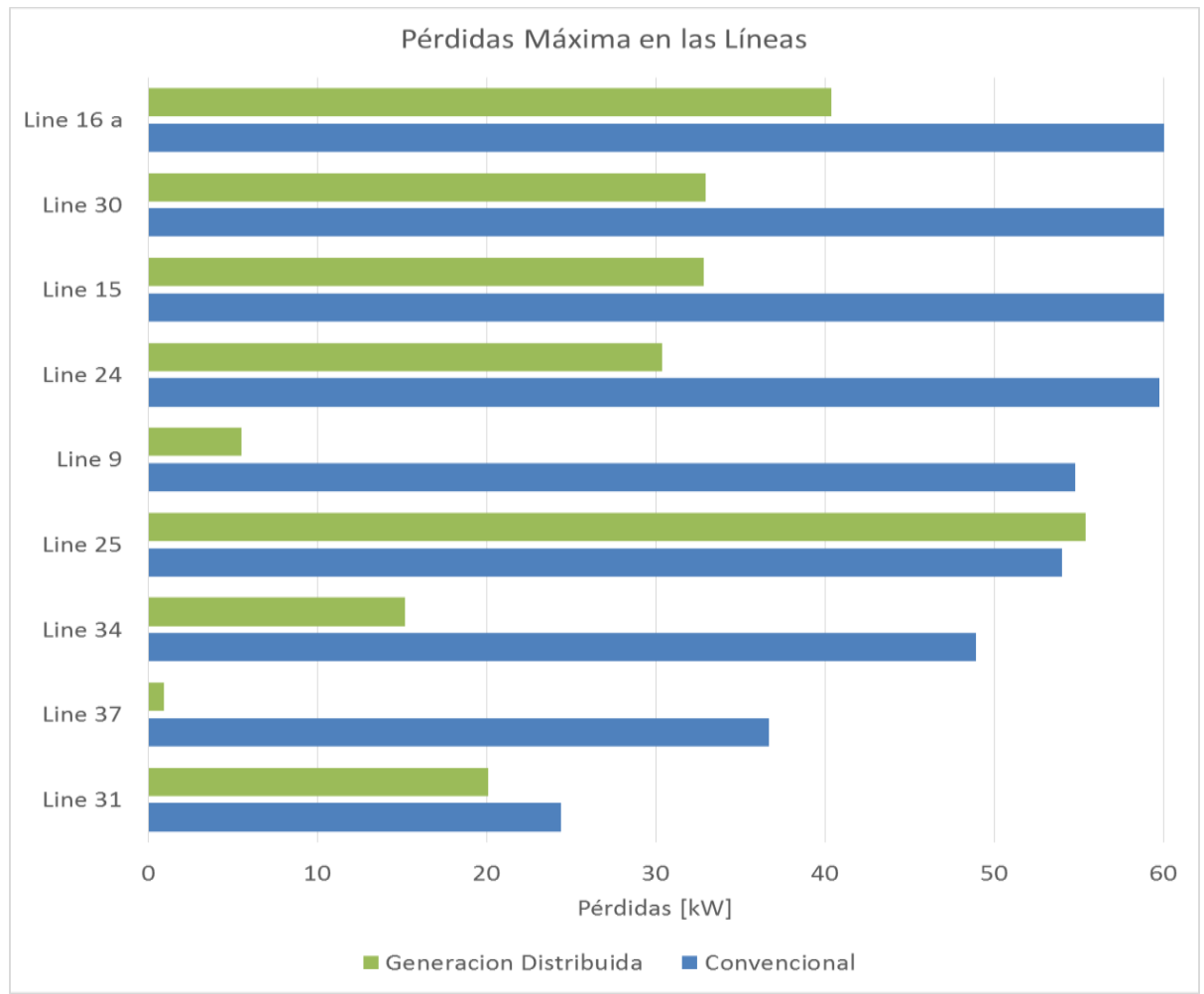

Figura 13. Valores de pérdidas en algunas líneas del sistema. Fuente: elaboración propia. 


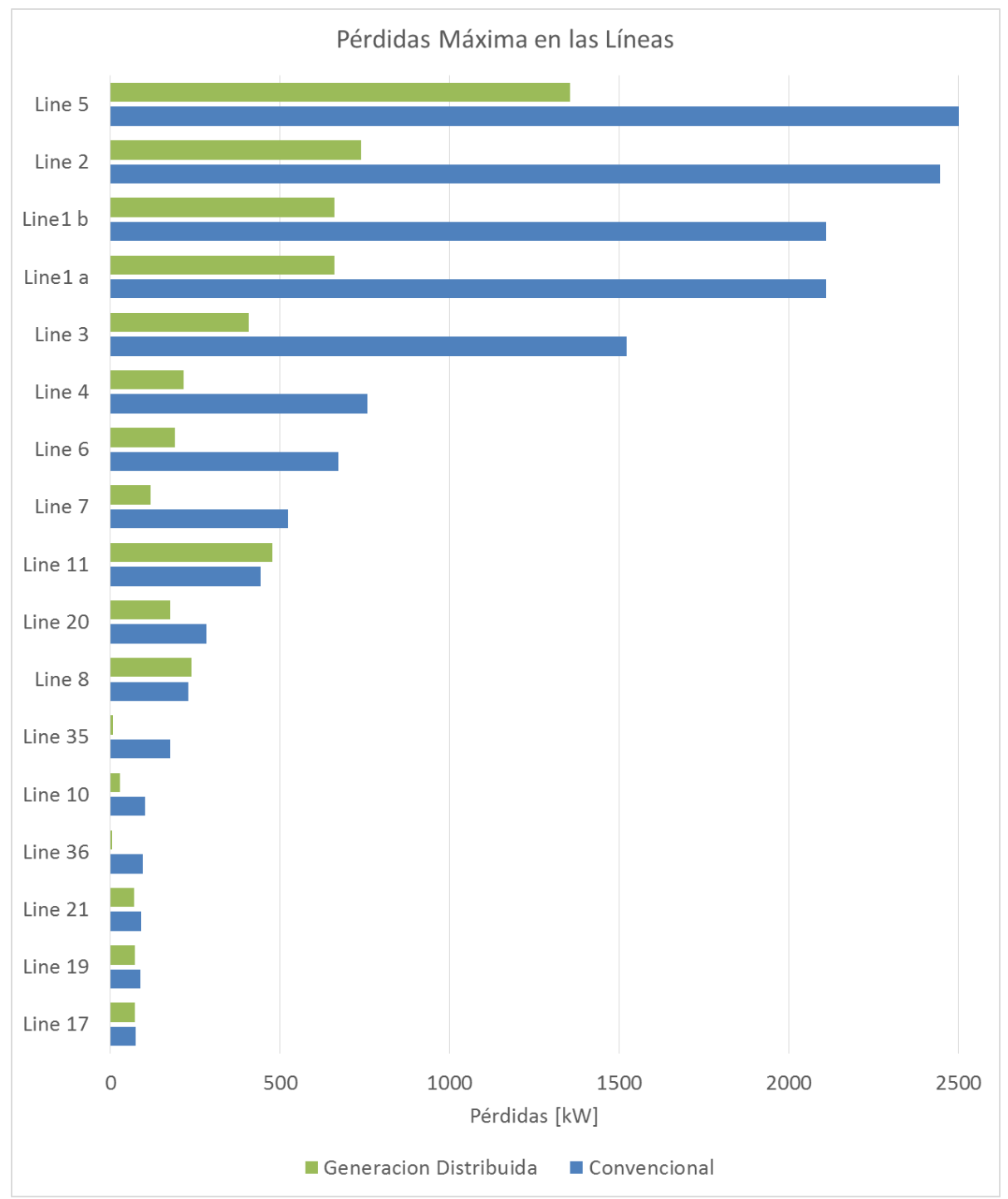

Figura 14. Valores de pérdidas en algunas líneas del sistema. Fuente: elaboración propia.

\section{Conclusiones}

Los resultados obtenidos para un escenario de simulación con un tipo de generación distribuida muestran que estos generadores no regulan la tensión en la red. La causa del incremento de los niveles de tensión se debe a la disminución en la potencia activa en algunos nodos, como consecuencia de la entrada en operación de los nodos que contienen GD en el sistema. Además, se observó que la inclusión de GD en el sistema trae una reducción considerable en la generación convencional, teniendo en cuenta que la GD no siempre puede trabajar a plena carga debido su carácter aleatorio.

La manera en que los nodos en los que están presentes los nuevos sistemas de generación suplen la demanda de las cargas presentes en el sistema IEEE de 30 nodos ocasiona que el nivel de corriente presente en la mayoría de líneas y transformadores del sistema disminuya.

Pasar de tener presentes en la red, nodos PQ a nodos PV (nodos GD) ocasionas que estos no estén en la capacidad de aportar reactivos al sistema. Para esta compensación se hace necesaria la entrada de tecnologías compensadoras de reactivos en el sistema de potencia.

En el escenario generación distribuida se observa que la entrada de generación distribuida al sistema se traduce en una disminución en las pérdidas presentes en la mayoria de líneas y transformadores del sistema. En este escenario de simulación se tiene una disminución en las pérdidas de las líneas de hasta un $97,35 \%$. Las pérdidas en los transformadores del sistema disminuyen hasta un $70,15 \%$. 
Las líneas que, por el contrario, presentan un aumento significativo en las pérdidas son las líneas 29 y 32. Este aumento excesivo en las pérdidas de las líneas se debe a que, por la ubicación de los nuevos generadores, las líneas pasan de transportar 40 a 120 y 10 a 60 amperios respectivamente, lo que influye directamente en las pérdidas presentes en esas líneas. En este caso se hace necesario hacer un estudio más profundo para concluir si estas líneas están en la capacidad de soportar ese nivel de corriente sin afectar su correcta operación.

En ese orden de ideas, los cambios en los flujos de corriente de este sistema, al estar presentes las nuevas tecnologías de generación en los nodos del sistema, son la causa de esto, ya que existe un aumento de las corrientes presentes en las líneas, lo que ocasiona el aumento en las pérdidas.

Los transformadores presentes en el sistema cumplen originalmente la función de adecuar el nivel de tensión de los nodos de generación convencional para suplir la demanda de las cargas. Al entrar nuevos generadores que suplen la demanda de ciertos nodos del sistema, la demanda total de las cargas presentes en el sistema disminuye, lo que ocasiona que los valores de perdidas en los transformadores disminuyan en la mayoria de ellos, ya que las corrientes presentes son menores a las de los sistemas originales.

Un estudio previo de ubicación óptima de GD en los sistemas, cuyo objetivo sea determinar la ubicación y el tamaño de múltiples fuentes de GD y minimizar las pérdidas de potencia totales del sistema, permitiría aumentar las ventajas de la inclusión de GD en el sistema, y restringiría la cantidad de líneas que presentaron un aumento en el nivel de pérdidas.

\section{Agradecimientos}

Los autores quieren agradecer a los docentes del Grupo de Compatibilidad Electromagnetica (GCEM) de la Facultad de Ingenieria de la Universidad Distrital, por sus asesoría en el desarrollo de este trabajo. A la rama IEEE de la Universidad Distrital, ya que a través de su Semillero de Investigación apoyó el desarrollo y la difusión de este trabajo dentro de la Universidad Distrital. Al ingeniero Carlos Andrés Velásquez Jiménez, por su colaboración en el transcurso de la investigación. L. Gaitan y J. Gómez agradecen a Ingeniería Especializada S. A., empresa que nos facilitó continuar con este trabajo de investigación mientras trabajaban para ellos.

\section{Referencias}

[1] G. A. C. Godoy, Impacto de la generación distribuida en la operación de la distribución, Santiago de Chile: Universidad de Chile, Facultad de Ciencias Físicas Y Matemáticas, Departamento De Ingeniería Eléctrica, 2013.

[2] A. F. Sarabia, Impact of distributed generation on distribution system, Aalborg: Department of Energy Technology, Faculty of Engineering, Science and Medicine, Aalborg University, 2011.

[3] Congreso de Colombia, «Ley 1715 de 2014: Por medio de la cual se regula la integración de las energías renovables no convencionales al Sistema Energético Nacional.,» 2014.

[4] Power, «Electric power systems analysis \& natureinspires optimization algorithms,» [En línea]. Available: http://www.al-roomi.org/power-flow.

[5]D. M. Escobar, Formulación del Despacho Económico en el Mercado de Energía con Alta Penetración de Energía Eólica, Guadalajara: Centro de Investigación y de Estudios Avanzados del Instituto Politécnico Nacional Unidad Guadalajara, 2016.

[6]Agosto 2016. [En línea]. Available: http://www.alroomi.org/power-flow.

[7]Instituto Colombiano de Normas Técnicas y Certificación, Icontec, NTC 819, Norma Tecnica Colombiana 819, Electrotecnia. Transformadores trifásicos autorefrigerados y sumergidos en liquido. corriente sin carga, pérdidas y tension de cortocircuito., Icontec, 1995.

[8]S. Castaño, Redes de distribución de energía, Manizales: Universidad Nacional de Colombia, 2009.

[9]A. Pedraza, D. Reyes, C. Gómez y F. Santamaría, «Impacto de la Generación Distribuida sobre el Esquema de Protecciones en una Red de Distribució,» Universidad Distrital Francisco Jose de Caldas, 2015.

[10]GmbH DigSilent, DigSilent PowerFactory Version 15 User Manual, 2014.

[11]A. F. P. Hernández, Localización de fallas en alimentadores primarios de distribución de energía eléctrica considerando incertidumbres en la carga y con presencia de Generación Distribuida, Pereira: Universidad Tecnológica De Pereira, 2013. 
[12] J. F. Schutte, Particle Swarms in sizing and global optimization, University of Pretoria, 2001.

[13] E. R. y K. J., «Particle Swarm Optimization,» de Proceedings of IEEE International Conference on Neural Networks Vol. IV: 1942 - 1948, 1995.

[14] B. Birge, «Mathworks,» 2006. [En línea]. Available:

[15] T. Rambharose, «Mathworks,» Noviembre 2010. [En línea]. Available: https://www.mathworks.com/matlabcentral/fileexchang e/29565-neural-network-add-in-for-psort.

[16] R. Danaraj, «Mathwoks,» Marzo 2014. [En línea]. Available:

https://www.mathworks.com/matlabcentral/fileexchang e/46002-optimal-power-flow-by-vector-pso.

[17] R. Danaraj, «Mathworks,» Agosto 2008. [En línea]. Available:

https://www.mathworks.com/matlabcentral/fileexchang e/20984-pso-solution-to-economic-dispatch.

[18] R. Danaraj, «Mathworks,» Marzo 2009. [En línea]. Available:

https://www.mathworks.com/matlabcentral/fileexchang e/23491-improved-pso-program-to-solve-economicdispatch.

[19]Mathworks, «Mathworks,» Diciembre 2009. [En línea]. Available: https://www.mathworks.com/matlabcentral/fileexchang e/25986-constrained-particle-swarm-optimization.

[20]S. Frank y S. Rebennack, A primer on optimal power flow: Theory, Formulation, and practical examples, Golden, 2012.

[21]J. Nuñez, Comparacion tecnica entre los programas de simulacion de sistemas de potencia DigSilent PowerFactory y PSSS/E, 2015.

[22] F.A. Zúñiga-Cortés, E.F. Caicedo-Bravo and D.M López-Santiago, "Gestión óptima de la potencia eléctrica en una microrred conectada, basada en el algoritmo genético para optimización multiobjetivo Moga," Rev. UIS Ing., vol. 15, no. 2, pp. 17-33, 2016. DOI: 10.18273/revuin.v15n2-2016002.

[23] V. Toro, S. Rivera and E. Mojica-Nava, "Mejoras de la regulación de frecuencia utilizando el aumento de inercia de microrredes interconectadas," Rev. UIS Ing., vol. 16, no. 2, pp. 35-42, 2017. DOI: https://doi.org/10.18273/revuin.v16n2-2017003 